\title{
On-the-fly Uniformization of Time-Inhomogeneous Infinite Markov Population Models
}

\author{
Aleksandr Andreychenko \\ Pepijn Crouzen \\ Linar Mikeev \\ Verena Wolf \\ Saarland University \\ Saarbrücken, Germany \\ \{aleand, crouzen, mikeev, wolf\}ecs.uni-saarland.de
}

\begin{abstract}
This paper presents an on-the-fly uniformization technique for the analysis of time-inhomogeneous Markov population models. This technique is applicable to models with infinite state spaces and unbounded rates, which are, for instance, encountered in the realm of biochemical reaction networks. To deal with the infinite state space, we dynamically maintain a finite subset of the states where most of the probability mass is located. This approach yields an under-approximation of the original, infinite system. We present experimental results to show the applicability of our technique.
\end{abstract}

\section{Introduction}

Markov population models (MPMs) are continuous-time Markov processes, where the state of the system is a vector of natural numbers (i.e., the populations). Such models are used in various application domains: biology, where the state variables describe the population sizes of different organisms, queueing theory, where we model a state as a vector of queue occupancies, chemistry, where the state variables represent the amount of molecules of different chemical species, etc [11].

Besides the expectations and variances of the different populations, the probabilities of certain events occurring can be of interest when studying MPMs. It may be necessary to know the probability of the extinction of a species, the probability that a population reaches a certain threshold, or even the full distribution of the MPM at a certain time-point, for instance to calibrate model parameters.

Many Markov population models have infinitely many states. In the case of biological or chemical applications, we normally cannot provide hard upper bounds for population numbers and in the field of queueing theory it may be interesting to consider unbounded queues. The evaluation of infinite MPMs through numerical [4] or statistical [7] analysis has been well-studied for time-homogeneous models where the dynamics of the system are independent of time. In [4] the state space of the model is generated and truncated on-the-fly during the transient solution, that is, during a certain time interval only states that are relevant at that time are considered. Thus, states are added at a certain step and dropped at a later time when they become irrelevant. A similar technique is proposed in [3] for the solution of timehomogeneous discrete-time Markov chains. Note that this is different from on-the-fly techniques for the computation of steady-state probabilities where the relevent part of the state space is generated but states are never dropped as time progresses [14].

Many Markov models are time-inhomogeneous, that is, their dynamics change over time. For instance, when modeling an epidemic, we may have to take into account that infection rates vary seasonally. For traffic models, time-dependent arrival rates can be used to model the morning and evening rush hours. In cellular biology we see that reaction propensities depend on the cell volume, which waxes and wanes as the cell grows and divides. The class of finite time-inhomogeneous Markov models has also been studied in recent years [2, 5, 13].

M. Massink and G. Norman (Eds.): 9th Workshop on

Quantitative Aspects of Programming Languages (QAPL 2011)

EPTCS 57, 2011, pp. 1-15 doi 10.4204/EPTCS.57.1 
In this paper, we develop a numerical algorithm to approximate transient probability distributions (i.e., the probability to be in a certain state at a certain time) for infinite time-inhomogeneous MPMs. We consider MPMs with state-dependent rates and do not require the existence of an upper-bound for the transition rates in the MPM.

Our algorithm is based on the uniformization technique, which is a well-known method to approximate the transient probability distribution of finite time-homogeneous Markov models [10, 9]. Recently, two adaptations of uniformization have been developed. These adaptations respectively approximate the transient probabilities for finite time-inhomogeneous [2] and infinite time-homogeneous [4] Markov models. Our algorithm combines and refines these two techniques such that infinite time-inhomogeneous MPMs with unbounded rates can be tackled. We present two case studies to investigate the effectiveness of our approach.

\section{Markov Population Models}

Markov chains with large or even infinite state spaces are usually described by some high-level modeling formalism that allows the generation of a (possibly infinite) set of states and transitions. Here, we use transition classes to specify a Markov population model, that is, a continuous-time Markov chain (CTMC) $\{X(t), t \geq 0\}$ with state space $S=\mathbb{Z}_{+}^{n}=\{0,1, \ldots\}^{n}$, where the $i$-th state variable represents the number of instances of the $i$-th species. Depending on the application area, "species" stands for types of system components, molecules, customers, etc. The application areas that we have in mind are chemical reaction networks, performance evaluation of computer systems, logistics, epidemics, etc [11].

Definition 1 (Transition Class) A transition class $\tau$ is a triple $(G, w, \alpha)$ where $G \subseteq \mathbb{Z}_{+}^{n}$ is the guard, $w \in \mathbb{Z}^{n}$ is the change vector, and $\alpha: G \times \mathbb{R}_{\geq 0} \rightarrow \mathbb{R}_{\geq 0}$ is the time-dependent rate function. Moreover, for any $x \in \mathbb{Z}_{+}^{n}$, we have that $x \in G$ implies $x+w \in \mathbb{Z}_{+}^{n}$.

The guard is the set of states where an instance of $\tau$ is possible, and if the current state is $x \in G$ then $x+w \in \mathbb{Z}_{+}^{n}$ is the state after an instance of $\tau$ has occurred. The rate $\alpha(x, t)$ determines the time-dependent transition probabilities for an infinitesimal time-step $d t$

$$
\operatorname{Pr}(X(t+d t)=x+w \mid X(t)=x)=\alpha(x, t) \cdot d t+o(d t),
$$

where $o$ is a function such that $o(0)=0$ and $\lim _{h \rightarrow 0} o(h) / h=0$.

A CTMC $X$ can be specified by a set of $m$ transition classes $\tau_{1}, \ldots, \tau_{m}$ as follows. For $j \in\{1, \ldots, m\}$, let $\tau_{j}=\left(G_{j}, w_{j}, \alpha_{j}\right)$. For each $t \in \mathbb{R}_{\geq 0}$ we define the generator matrix $Q(t)$ of $X$ such that the row that describes the transitions of a state $x$ has entry $\alpha_{j}(x, t)$ at position $Q(t)_{x, x+w_{j}}$ whenever $x \in G_{j}$ and zero otherwise. Moreover, the diagonal entries of $Q(t)$ are the negative sums of the off-diagonal row entries because the row sums of a generator matrix are zero. We assume that each change vector $w_{j}$ has at least one non-zero entry. To simplify the presentation we assume that all change vectors are distinct. We remark that $X$ is called time-homogeneous when $Q(t)$ is equal for all $t$. Otherwise, $X$ is called timeinhomogeneous.

Example 1 We consider a simple gene expression model for E. coli cells [16]. It consists of the transcription of a gene into messenger RNA ( $m R N A$ ) and subsequent translation of the latter into proteins. A state of the system is uniquely determined by the number of $m R N A$ and protein molecules, that is, a state is a pair $\left(x_{M}, x_{P}\right) \in \mathbb{Z}_{+}^{2}$. We assume that initially there are no $m R N A$ molecules and no proteins in the system, i.e., $\operatorname{Pr}(X(0)=(0,0))=1$. Four types of reactions occur in the system. Let $j \in\{1, \ldots, 4\}$ and 
$\tau_{j}=\left(G_{j}, w_{j}, \alpha_{j}\right)$ be the transition class that describes the $j$-th reaction type. We first define the guard sets $G_{1}, \ldots, G_{4}$ and the change vectors $w_{1}, \ldots, w_{4}$.

- Transition class $\tau_{1}$ models gene transcription. The corresponding stoichiometric equation is $\emptyset \rightarrow$ $m R N A$. If a $\tau_{1}$-transition occurs, the number of $m R N A$ molecules increases by one. Thus, $w_{1}=$ $(1,0)$. This transition class is possible in all states, i.e., $G_{1}=\mathbb{Z}_{+}^{2}$.

- We represent the translation of $m R N A$ into protein by $\tau_{2}(m R N A \rightarrow m R N A+P)$. A $\tau_{2}$-transition is only possible if there is at least one mRNA molecule in the system. We set $G_{2}=\left\{\left(x_{M}, x_{P}\right) \in \mathbb{Z}_{+}^{2} \mid\right.$ $\left.x_{R}>0\right\}$ and $w_{2}=(0,1)$. Note that in this case $m R N A$ is a reactant that is not consumed.

- Both mRNA and protein molecules can degrade, which is modelled by $\tau_{3}$ and $\tau_{4}$ (mRNA $\rightarrow \emptyset$ and $P \rightarrow \emptyset)$. Hence, $G_{3}=G_{2}, G_{4}=\left\{\left(x_{M}, x_{P}\right) \in \mathbb{Z}_{+}^{2} \mid x_{P}>0\right\}$, $w_{3}=(-1,0)$, and $w_{4}=(0,-1)$.

Let $k_{1}, k_{2}, k_{3}, k_{4}$ be real-valued positive constants. We assume that transcription happens at rate $\alpha_{1}\left(x_{M}, x_{P}, t\right)=k_{1} \cdot V(t)$, that is, the rate is proportional to the cell volume $V(t)$ [17]. The (timeindependent) translation rate depends linearly on the number of $m R N A$ molecules. Therefore, $\alpha_{2}\left(x_{M}, x_{P}, t\right)$ $=k_{2} \cdot x_{M}$. Finally, for degradation, we set $\alpha_{3}\left(x_{M}, x_{P}, t\right)=k_{3} \cdot x_{M}$ and $\alpha_{4}\left(x_{M}, x_{P}, t\right)=k_{4} \cdot x_{P}$.

We now discuss the transient probability distribution of a MPM. Let $S$ be the state space of $X$ and let the transition function $P(t, t+\Delta)$ be such that the entry for the pair $(x, y)$ of states equals

$$
P(t, t+\Delta)_{x y}=\operatorname{Pr}(X(t+\Delta)=y \mid X(t)=x), \quad t, \Delta \geq 0 .
$$

If the initial probabilities $\operatorname{Pr}(X(0)=x)$ are specified for each $x \in S$, the transient state probabilities $p^{(t)}(x):=\operatorname{Pr}(X(t)=x)$, are given by

$$
p^{(t)}(y)=\sum_{x \in S} p^{(0)}(x) \cdot P(0, t)_{x y} .
$$

We assume that a transition class description uniquely specifies a CTMC and rule out "pathological cases" by assuming that the sample paths $X(t)$ are right-continuous step functions. In this case the transition functions are the unique solution of the Kolmogorov backward and forward equations

$$
\begin{aligned}
& \frac{d}{d t} P\left(t_{0}, t\right)=Q(t) \cdot P\left(t_{0}, t\right) \\
& \frac{d}{d t} P\left(t_{0}, t\right)=P\left(t_{0}, t\right) \cdot Q(t),
\end{aligned}
$$

where $0 \leq t_{0} \leq t$. Multiplication of Eq. (2) with the row vector $p^{\left(t_{0}\right)}$ with entries $p^{\left(t_{0}\right)}(x)$ gives

$$
\frac{d}{d t} p^{(t)}=p^{(t)} \cdot Q(t)
$$

If $S$ is finite, algorithms for the computation of $p^{(t)}$ are usually based on the numerical integration of the linear system of differential equations in Eq. (3) with initial condition $p^{(0)}$. Here, we focus on another approach called uniformization that is widely used for time-homogeneous Markov chains [10]. It has been adapted for time-inhomogeneous Markov chains by Van Dijk [5] and subsequently improved [13, 2]. The main advantage of solution techniques based on uniformization is that they provide an underapproximation of the vector $p^{(t)}$ and, thus, provide tight error bounds. Moreover, they are numerically stable and often superior to numerical integration methods in terms of running times [15]. 


\section{Uniformization}

Uniformization is based on the idea to construct, for a CTMC $X$, a Poisson process $N(t), t \geq 0$ and a subordinated discrete-time Markov chain (DTMC) $Y(i), i \in \mathbb{N}$ such that for all $x$ and for all $t$

$$
\operatorname{Pr}(X(t)=x)=\operatorname{Pr}(Y(N(t))=x) .
$$

Since Poisson process $N$ and DTMC $Y$ are independent, the equation above can be written as

$$
\operatorname{Pr}(Y(N(t))=x)=\sum_{i=0}^{\infty} \operatorname{Pr}(Y(i)=x) \operatorname{Pr}(N(t)=i) .
$$

For a finite time-homogeneous MPM with state space $S$ the rate $\Lambda$ of the Poisson process $N$ (also called the uniformization rate) is chosen to be greater than or equal to the maximal exit-rate appearing in $X$

$$
\Lambda \geq \max _{x \in S} \sum_{j=1}^{m} \alpha_{j}(x)
$$

For the DTMC $Y$ we find transition probabilities

$$
\operatorname{Pr}\left(Y(i+1)=x+w_{j} \mid Y(i)=x\right)=\frac{\alpha_{j}(x)}{\Lambda} .
$$

When $X$ is time-inhomogeneous, Arns et al. [2] suggest to define the time-dependent uniformization rate $\Lambda(t)$ of the inhomogeneous Poisson process (IPP) $N$ as

$$
\Lambda(t) \geq \max _{x \in S} \sum_{j=1}^{m} \alpha_{j}(x, t) .
$$

For the (time-dependent) transition probabilities of the DTMC $Y$ we then have that $\frac{\alpha_{j}(x, t)}{\Lambda(t)}$ is the probability to enter state $x+w_{j}$ from state $x$ if a state-change occurs at time $t$. Arns et al. prove that Eq. (4) holds if the $\alpha_{j}$ are (right or left) continuous functions in $t$ and if $S$ is finite (see Theorem 7 in [2]). Here, we relax the latter condition and allow $S$ to be infinite. If $\sup _{x \in S} \sum_{j} \alpha_{j}(x, t)<\infty$ during the time interval of interest, the proof of Eq. (4) may be expected to proceed along similar lines. In our case, however, $\sup _{x \in S} \sum_{j} \alpha_{j}(x, t)=\infty$ and then the Poisson process $N$ is not well-defined as its rate must be infinite according to Eq. (6). Therefore, the infinite state space has to be truncated in an appropriate way.

\subsection{State Space Truncation}

We consider a time interval $[t, t+\Delta)$ of length $\Delta$, where the transient distribution at time $t, p^{(t)}$, of the infinite time-inhomogeneous MPM $X$ is known. We now wish to approximate the transient distribution at time $t+\Delta, p^{(t+\Delta)}$. We assume that $p^{(t)}$ has finite support $S_{t, 0}$. Define $\operatorname{Pr}(N(t, t+\Delta)=i)=$ $\operatorname{Pr}(N(t+\Delta)-N(t)=i)$ as the probability that $N$ performs $i$ steps within $[t, t+\Delta)$. For a fixed positive $\varepsilon \ll 1$, let $R$ and the rate function $\Lambda$ be such that $S_{t, R}$ is the set of states that are reachable, with probability greater than or equal to $1-\varepsilon$, from the set $S_{t, 0}$ in the time-interval $[t, t+\Delta)$ within at most $R$ transitions, i.e.

$$
\sum_{i=0}^{R} \operatorname{Pr}(N(t, t+\Delta)=i) \geq 1-\varepsilon
$$


Furthermore, we have that the rate of $N$ at time $t^{\prime} \in[t, t+\Delta)$ must satisfy

$$
\Lambda\left(t^{\prime}\right) \geq \max _{x \in S_{t, R}} \sum_{j=1}^{m} \alpha_{j}\left(x, t^{\prime}\right)
$$

Note that $\Lambda\left(t^{\prime}\right)$ is adaptive and depends on $t^{\prime}, t, \Delta, S_{t, 0}$, and $R$ as opposed to Arns et al. where $\Lambda\left(t^{\prime}\right)$ depends only on $t^{\prime}, t$, and $\Delta$ because they consider finite state spaces.

Finding appropriate values for $\Delta$ and $R$ is non-trivial as $\Lambda\left(t^{\prime}\right)$ determines the speed of the Poisson process $N$ and thereby influences the value of $R$. On the other hand, $R$ determines the size of the set $S_{t, R}$ and thus influences $\Lambda\left(t^{\prime}\right)$. We discuss how to find appropriate choices for $\Delta$ and $R$ given the set $S_{t, 0}$ in Section 4.1

Assume that we find $\Delta$ and $R$ with the above mentioned properties and define $\Lambda\left(t^{\prime}\right)$ as in Eq. (8). Then, for all $x \in S$, we get an $\varepsilon$-approximation

$$
\operatorname{Pr}(X(t+\Delta)=x) \geq \sum_{i=0}^{R} \operatorname{Pr}(Y(i)=x \wedge N(t, t+\Delta)=i),
$$

where $Y$ has initial distribution $p^{(t)}$. The probabilities $\operatorname{Pr}(Y(i)=x \wedge N(t, t+\Delta)=i)$ can now be approximated in the same way as for the finite case [2].

From Eq. (9) we see that it is beneficial if $R$ is small, since this means fewer probabilities have to be computed in the right-hand side of Eq. (9). Note that the truncation-point $R$ is small when the uniformization rates $\Lambda\left(t^{\prime}\right)$ are small during $[t, t+\Delta)$ because if $N$ jumps at a slower rate then $\operatorname{Pr}(N(t, t+\Delta)>i)$ becomes smaller. Thus, it is beneficial to choose $\Lambda\left(t^{\prime}\right)$ as small as possible while still satisfying Eq. (8).

\subsection{Bounding approach}

Let $\hat{p}^{(t+\Delta)}(x)$ denote the right hand side of Eq. (9), i.e., the approximation of the transient probability of state $x$ at time $t+\Delta$. We compute this approximation with the uniformization method as follows. The processes $Y$ and $N$ are independent which implies that

$$
\operatorname{Pr}(Y(i)=x \wedge N(t, t+\Delta)=i)=\operatorname{Pr}(Y(i)=x) \cdot \operatorname{Pr}(N(t, t+\Delta)=i) .
$$

The probabilities $\operatorname{Pr}(N(t, t+\Delta)=i)$ follow a Poisson distribution with parameter $\bar{\Lambda}(t, t+\Delta) \cdot \Delta$, where

$$
\bar{\Lambda}(t, t+\Delta)=\frac{1}{\Delta} \int_{t}^{t+\Delta} \Lambda\left(t^{\prime}\right) d t^{\prime} .
$$

For the distribution $\operatorname{Pr}(Y(i)=x)$, Arns et al. suggest an underapproximation that relies on the fact that for any time-point $t^{\prime} \in[t, t+\Delta)$ we have:

$$
\frac{\alpha_{j}\left(x, t^{\prime}\right)}{\Lambda\left(t^{\prime}\right)} \geq \min _{t^{\prime \prime} \in[t, t+\Delta]} \frac{\alpha_{j}\left(x, t^{\prime \prime}\right)}{\Lambda\left(t^{\prime \prime}\right)}=: u_{j}(x, t, t+\Delta) .
$$

Thus, for $i \in\{1,2, \ldots, R\}$, we iteratively approximate $\operatorname{Pr}(Y(i)=y)$ as

$$
\operatorname{Pr}(Y(i)=y) \geq \sum_{x, j: y=x+w_{j}} \operatorname{Pr}(Y(i-1)=x) \cdot u_{j}(x, t, t+\Delta)+\operatorname{Pr}(Y(i-1)=y) \cdot u_{0}(y, t, t+\Delta) .
$$

Here, $x$ ranges over all direct predecessors of $y$ and the self-loop probability $u_{0}(y, t, t+\Delta)$ of $y$ is given by

$$
u_{0}(y, t, t+\Delta)=\min _{t^{\prime} \in[t, t+\Delta]}\left(1-\sum_{j=1}^{m} \frac{\alpha_{j}\left(y, t^{\prime}\right)}{\Lambda\left(t^{\prime}\right)}\right) .
$$


Note that often we can split $\alpha_{j}\left(x, t^{\prime}\right)$ into two factors $\lambda_{j}\left(t^{\prime}\right)$ and $r_{j}(x)$ such that $\alpha_{j}\left(x, t^{\prime}\right)=\lambda_{j}\left(t^{\prime}\right) \cdot r_{j}(x)$ for all $t^{\prime}, j, \sqrt{1}$. Thus, the functions $\lambda_{j}: \mathbb{R}_{\geq 0} \rightarrow \mathbb{R}_{>0}$ contain the time-dependent part (but are stateindependent) and the functions $r_{j}: S \rightarrow \mathbb{R}_{>0}$ contain the state-dependent part (but are time-independent). Then each minimum defined above can be computed for all states by considering

$$
\min _{t^{\prime} \in[t, t+\Delta]} \frac{\lambda_{j}\left(t^{\prime}\right)}{\Lambda\left(t^{\prime}\right)} .
$$

In particular, if $\lambda_{j}$ and $\Lambda$ are monotone, the above minimum is easily found analytically.

The approximation in Eq. (10) implies that for the time interval $[t, t+\Delta)$, we compute a sequence of substochastic vectors $v^{(1)}, v^{(2)}, \ldots, v^{(R)}$ to approximate the probabilities $\operatorname{Pr}(Y(i)=y)$. Initially we start the DTMC $Y$ with the approximation $\hat{p}^{(t)}=: v^{(0)}$ of the previous step. Then we compute $v^{(i+1)}$ from $v^{(i)}$ based on the transition probabilities $u_{j}(x, t, t+\Delta)$ for $i \in\{0,1, \ldots, R\}$. Since these transition probabilities may sum up to less than one, the resulting vector $v^{(i+1)}$ may also sum up to less than one. Since, for the computation of $\hat{p}^{t+\Delta}$, we weight these vectors with the Poisson probabilities and add them up the underapproximation $\hat{p}^{t+\Delta}$ contains an additional approximation error. In general, the larger the time-period $\Delta$, the worse the underapproximations $u_{j}(x, t, t+\Delta)$ are and thus the underapproximation $\hat{p}^{t+\Delta}$ becomes worse as well. We illustrate this effect by applying the bounding approach to our running example.

Example 2 In the gene expression of Example 1 the time-dependence is due to the volume and only affects the rate function $\alpha_{1}$ of the first transition class. The time until an E. coli cell divides varies widely from about 20 minutes to many hours and depends on growth conditions. Here, we assume a cell cycle time of one hour and a linear growth [1]. Thus, if at time $t=0$ we consider a cell immediately after division then the cell volume doubles after $3600 \mathrm{sec}$. Assume that $\Delta \leq 3600$. Then, $\alpha_{1}\left(x, t^{\prime}\right)=$ $k_{1}^{\prime} \cdot\left(1+\frac{t^{\prime}}{3600}\right)$ for all $x \in S$. Assume we have a right truncation point $R$ such that

$$
\Lambda\left(t^{\prime}\right)=\max _{x_{R}, x_{P}} k_{1}^{\prime} \cdot\left(1+\frac{t^{\prime}}{3600}\right)+\left(k_{2}+k_{3}\right) \cdot x_{R}+k_{4} \cdot x_{P}
$$

where $x_{R}$ and $x_{P}$ range over all states $\left(x_{R}, x_{P}\right) \in S_{0, R}$ and $E q$. (7) holds. Then we find, for each time-point $t^{\prime} \in[0, \Delta)$, the same state for which the exit-rate $\alpha_{0}\left(x, t^{\prime}\right):=\sum_{j=1}^{m} \alpha_{j}\left(x, t^{\prime}\right)$ is maximal, since the only timedependent propensity is independent of the state-variables. Let $\left(x_{R}^{\max }, x_{P}^{\max }\right)$ denote this state. In general this is not the case, for instance in the realm of chemical reaction systems we have that the propensities of bimolecular reactions (reactions of the from $A+B \rightarrow \ldots$ ) are dependent both on cell-volume and the population numbers. For such a system we may find that different states have the maximal exit-rate within the time-frame $[0, \Delta)$. We discuss how to overcome this difficulty in Subsection 4.2 The transition probabilities of the DTMC Y are now defined as

$$
u_{1}\left(x_{R}, x_{P}, 0, \Delta\right)=\min _{t^{\prime} \in[0, \Delta]} \frac{\alpha_{1}\left(x_{R}, x_{P}, t^{\prime}\right)}{\Lambda\left(t^{\prime}\right)}=\frac{\alpha_{1}(x, 0)}{\Lambda(0)}=\frac{k_{1}^{\prime}}{k_{1}^{\prime}+\left(k_{2}+k_{3}\right) \cdot x_{R}^{\max }+k_{4} \cdot x_{P}^{\max }}
$$

and, for $j \in\{2,3\}$,

$$
u_{j}\left(x_{R}, x_{P}, 0, \Delta\right)=\min _{t^{\prime} \in[0, \Delta]} \frac{\alpha_{j}\left(x_{R}, x_{P}, t^{\prime}\right)}{\Lambda\left(t^{\prime}\right)}=\min _{t^{\prime} \in[0, \Delta]} \frac{k_{j} \cdot x_{R}}{\Lambda(\Delta)}=\frac{k_{j} \cdot x_{R}}{k_{1}^{\prime} \cdot\left(1+\frac{\Delta}{3600}\right)+\left(k_{2}+k_{3}\right) \cdot x_{R}^{\max }+k_{4} \cdot x_{P}^{\max }},
$$

\footnotetext{
${ }^{1}$ Note that this decomposition is always possible for chemical reaction networks where the time-dependence stems from fluctuations in reaction volume or temperature.
} 

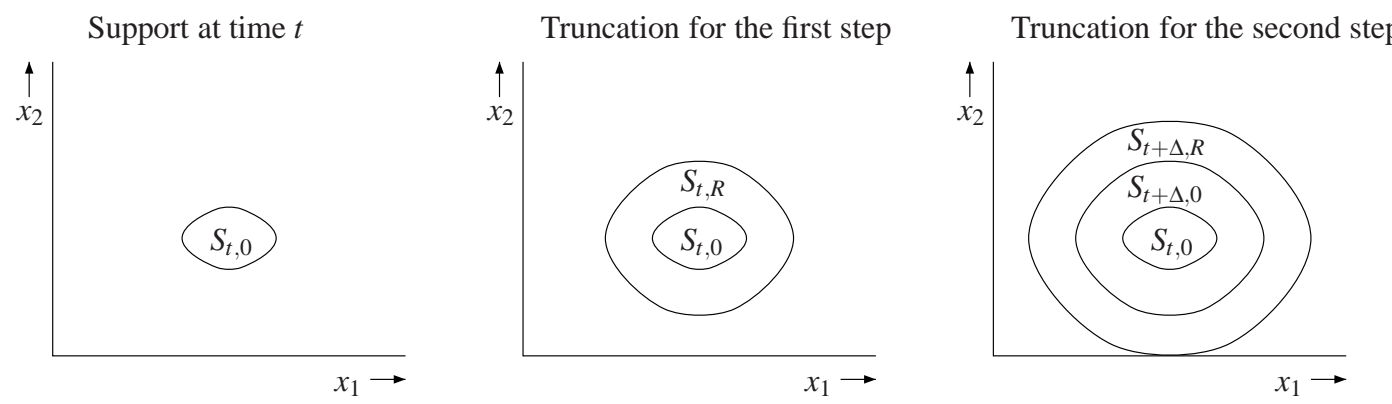

Figure 1: Illustration of the state space truncation approach for the two-dimensional case. Given the distribution $\hat{p}^{(t)}$ with support $S_{t, 0}$, a truncation point $R$ and a time-step $\Delta$, we compute in the first step the distribution $\hat{p}^{(t+\Delta)}$ with support $S_{t, R}=S_{t+\Delta, 0}$. For the next step we consider the set $S_{t+\Delta, R}$.

$$
u_{4}\left(x_{R}, x_{P}, 0, \Delta\right)=\frac{k_{4} \cdot x_{P}}{k_{1}^{\prime} \cdot\left(1+\frac{\Delta}{3600}\right)+\left(k_{2}+k_{3}\right) \cdot x_{R}^{\max }+k_{4} \cdot x_{P}^{\max }} .
$$

For the self-loop probability we find:

$$
\begin{gathered}
u_{0}\left(x_{R}, x_{P}, 0, \Delta\right)=\min _{t^{\prime} \in[0, \Delta]}\left(1-\sum_{j=1}^{4} \frac{\alpha_{j}\left(x_{R}, x_{P}, t^{\prime}\right)}{\Lambda\left(t^{\prime}\right)}\right)=\left(1-\max _{t^{\prime} \in[0, \Delta)} \sum_{j=1}^{4} \frac{\alpha_{j}\left(x_{R}, x_{P}, t^{\prime}\right)}{\Lambda\left(t^{\prime}\right)}\right) \\
=1-\sum_{j=1}^{4} \frac{\alpha_{j}\left(x_{R}, x_{P}, \Delta\right)}{\Lambda(\Delta)}=1-\frac{k_{1}^{\prime} \cdot\left(1+\frac{\Delta}{3600}\right)+\left(k_{2}+k_{3}\right) \cdot x_{R}+k_{4} \cdot x_{P}}{k_{1}^{\prime} \cdot\left(1+\frac{\Delta}{3600}\right)+\left(k_{2}+k_{3}\right) \cdot x_{R}^{\max }+k_{4} \cdot x_{P}^{\max }} .
\end{gathered}
$$

We now calculate the fraction of probability lost during the computation of $v^{(i+1)}$ from $v^{(i)}$, i.e.,

$$
\begin{gathered}
1-\sum_{j=0}^{4} u_{j}\left(x_{R}, x_{P}, 0, \Delta\right)=\frac{k_{1}^{\prime} \cdot\left(1+\frac{\Delta}{3600}\right)}{k_{1}^{\prime} \cdot\left(1+\frac{\Delta}{3600}\right)+\left(k_{2}+k_{3}\right) \cdot x_{R}^{\max }+k_{4} \cdot x_{P}^{\max }}-\frac{k_{1}^{\prime}}{k_{1}^{\prime}+\left(k_{2}+k_{3}\right) \cdot x_{R}^{\max }+k_{4} \cdot x_{P}^{\max }} \\
=\frac{\left(k_{2}+k_{3}\right) \cdot x_{R}^{\max }+k_{4} \cdot x_{P}^{\max }}{k_{1}^{\prime}+\left(k_{2}+k_{3}\right) \cdot x_{R}^{\max }+k_{4} \cdot x_{P}^{\max }}-\frac{\left(k_{2}+k_{3}\right) \cdot x_{R}^{\max }+k_{4} \cdot x_{P}^{\max }}{k_{1}^{\prime} \cdot\left(1+\frac{\Delta}{3600}\right)+\left(k_{2}+k_{3}\right) \cdot x_{R}^{\max }+k_{4} \cdot x_{P}^{\max }} .
\end{gathered}
$$

For $\Delta=0$ we have a probability loss of 0 and for $\Delta>0$ we can see that the probability loss increases with increasing $\Delta$.

\subsection{Time-stepping approach}

Given that a large time horizon may lead to decreased accuracy, Arns et al. [2] suggest to partition the time period of interest $\left[0, t_{\max }\right)$ in steps of length $\Delta$. In each step, an approximation of the transient distribution at the current time instant, $\hat{p}^{(t)}$, is computed and used as initial condition for the next step. The number of states that we consider, that is, $\left|S_{t, R}\right|$ grows in each step. The probabilities of all remaining states of $S$ are approximated as zero. Thus, each step yields a vector $\hat{p}^{(t+\Delta)}$ with positive entries for all states $x \in S_{t, R}$ that approximate $\operatorname{Pr}(X(t+\Delta)=x)$. The vector $\hat{p}^{(t+\Delta)}$ with support $S_{t, R}=S_{t+\Delta, 0}$ is then used as the initial distribution to approximate the vector $\hat{p}^{\left(t+\Delta+\Delta^{\prime}\right)}$. See Figure 1 for a sketch of the state truncation approach. Note that the chosen time-period $\Delta$ may vary for different steps of the approach. 
It is easy to see that the total error is the sum of the errors in each step, where the error of a single step equals the amount of probability mass that "got lost" due to the underapproximation. More precisely, we have two sources of error, namely the error due to the truncation of the infinite sum in Eq. (5) and the error due to the bounding approach that relies on Eq. (10).

In [2], Arns et al. give exact formulas for the first three terms of the sum in Eq. (9) (for $i=0,1,2$ ). Thus, if the approximation $\hat{p}^{(t)}$ of $p^{(t)}$ is exact, then $\hat{p}^{(t+\Delta)}$ is an underapproximation due to the remaining terms in Eq. (9). This implies that the smaller $R$ becomes, the closer the error will be to the error bound $\varepsilon$. On the other hand, a small truncation point means that only a small time step $\Delta$ is possible (see Eq. (7)), which means that many steps are necessary until the final time instant $t_{\max }$ is reached. In order to explore the trade-off between running time and accuracy, we run experiments with different values for the predefined truncation point $R$ that determines the step size $\Delta$. We report on these experiments in Section 5 .

\section{On-the-fly Algorithm}

As we can see in Figure 1 the number of states that are considered to compute $\hat{p}^{\left(t^{\max }\right)}$ from $\hat{p}^{(t)}$ grows in each step, since all states within a radius of $R$ transitions from a state in the previous set $S_{t, 0}$ are added. This makes the approach infeasible for Markov models with a large or even infinite state space because the memory requirements are too large. Therefore, we suggest to use a similar strategy as described in previous work [4] to keep the memory requirements low and achieve faster running times.

The underlying principle of this approach is to dynamically maintain a snapshot of the part of the state space where most of the transient probability distribution is located. We achieve this by adding and removing states in an on-the-fly fashion. The decision which states to add and which states to remove depends on a small probability threshold $\delta>0$. After the computation of the vector $v^{(i+1)}$ based on $v^{(i)}$, we set all entries in $v^{(i+1)}$ to zero that have a probability less than $\delta$. This significantly reduces the computational complexity since only parts of the transition probability matrix of $Y$ have to be generated [4] (for instance, we explore 360000 states at time instant $t=600$ for the gene expression system of Example 1 if $\delta=0$ but only 5700 states are stored when $\delta=10^{-15}$ ). Let

$$
S^{(0)}:=\left\{x: v^{(0)}(x)>0\right\}=S_{t, 0}
$$

and, for $i \in\{1, \ldots, R\}$ let $S^{(i)}$ be the set of states that we consider to compute $v^{(i+1)}$ from $v^{(i)}$. We remark that this also decreases the speed of the Poisson process $N$ since the sets $S_{t, 0}$ and $S_{t, R}$ are smaller and thus the maximum in Eq. (8) is now taken over fewer states. We illustrate this effect in Figure 2] This effect is particularly important if during an interval $\left[t, t^{\max }\right)$ in certain parts of the state space the dynamics of the system is fast while it is slow in other parts where the latter contain the main part of the probability mass. On the other hand, the threshold $\delta$ introduces another approximation error which may become large if the time horizon of interest is long. Moreover, if $\rho$ is a bound for the error introduced by the above strategy of neglecting certain states, we can reserve a portion of the probability loss $\rho \cdot \frac{\Delta}{t_{\max }}$ for the interval $[t, t+\Delta)$ and repeat the computation with a smaller threshold $\delta$ if more than the allowed portion of probability was neglected.

The approximation that we suggest above is again an underapproximation and since the approximations suggested in the previous sections are also underapproximations, we are still able to compute the total error of the approximation $\hat{p}^{(t)}$ of $p^{(t)}$ as

$$
1-\sum_{x \in S_{t, R}} \hat{p}^{(t)}(x)
$$



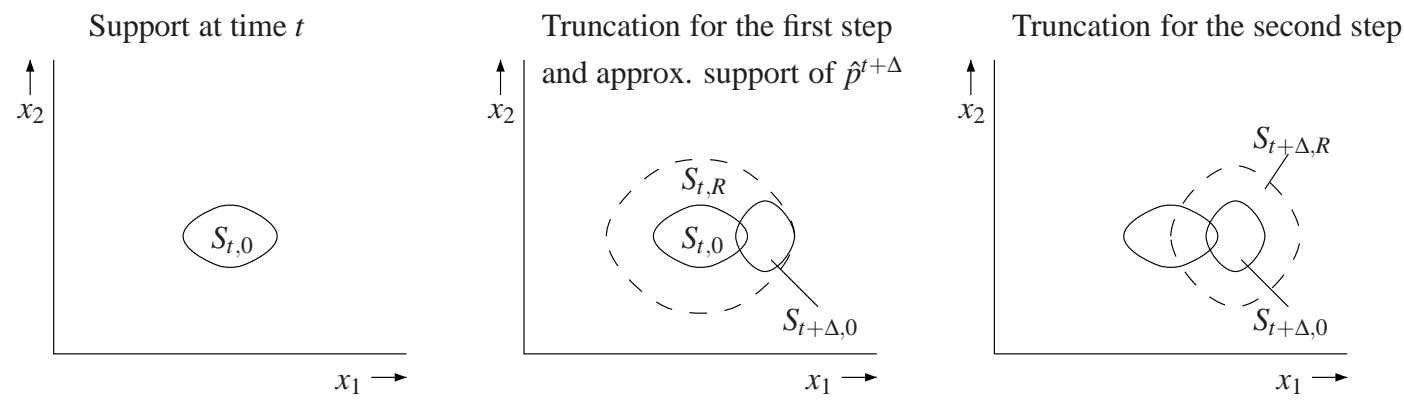

Figure 2: Illustration of the on-the-fly algorithm for the two-dimensional case. Given the distribution $\hat{p}^{(t)}$ with support $S_{t, 0}$, a truncation point $R$ and a time-step $\Delta$, we compute in the first step the distribution $\hat{p}^{(t+\Delta)}$ with approximate support $S_{t+\Delta, 0} \subset S_{t, R}$. For the next step we consider the set $S_{t+\Delta, R}$.

Clearly, $t^{\prime}>t$ implies that the error at time $t^{\prime}$ is higher than the error at time $t$. For our experimental results in Section 5 we choose $\delta=10^{-15}$ and report on the total error of the approximation at time $t_{\max }$.

\subsection{Determining the step-size}

Given an error bound $\varepsilon>0$, a time-point $t$, for which the support of $\hat{p}^{(t)}$ is $S_{t, 0}$, and a time-point $t_{\max }$ for which we wish to approximate the transient probability distribution, we now discuss how to find a timestep $\Delta$ such that Eqs. (7) and (8) hold. Recall that the probabilities $\operatorname{Pr}(N(t, t+\Delta)=i)$ follow a Poisson distribution with parameter $\bar{\Lambda}(t, t+\Delta) \cdot \Delta$, which we denote by $\mu_{R, \Delta}$ to emphasize the dependence on $\Delta$ and the right truncation point $R$. Note that the latter dependence is due to the maximum in Eq. (8) that is defined over the set $S_{t, R}$, the set of all states that are reachable from a state in $S_{t, 0}$ by at most $R$ transitions. We have

$$
\mu_{R, \Delta}=\int_{t}^{t+\Delta} \Lambda\left(t^{\prime}\right) d t^{\prime}
$$

Here, we propose to first choose a desired right truncation point $R^{*}$ and then find a time-step $\Delta$ such that Eqs. (7) and (8) hold. We perform an iteration where in each step we systematically choose different values for $\Delta$ and compare the associated right truncation point $R$ with $R^{*}$. Since $\mu_{R^{*}, \Delta}$ is monotone in $\Delta$ this can be done in a binary search fashion as described in Algorithm 1(a). We start with the two bounds $\Delta^{-}=0$ and $\Delta^{+}=t_{\max }-t$. The function FindMaxState $\left(\Delta, R^{*}\right)$ finds a state $x^{\max }$ such that for all time-points $t^{\prime} \in[t, t+\Delta)$ we have

$$
\sum_{j=1}^{m} \alpha_{j}\left(x^{\max }, t^{\prime}\right) \geq \max _{x^{\prime} \in S_{t, R^{*}}} \sum_{j=1}^{m} \alpha_{j}\left(x^{\prime}, t^{\prime}\right)
$$

The choice of $x^{\max }$ also determines the uniformization rate

$$
\Lambda\left(t^{\prime}\right)=\sum_{j=1}^{m} \alpha_{j}\left(x^{\max }, t^{\prime}\right)
$$

It immediately follows from Eq. (13) that Eq. (8) holds. In Section 4.2, we discuss how to find $\Lambda$ efficiently by selecting a state $x^{\text {max }}$, while avoiding that the uniformization rates $\Lambda\left(t^{\prime}\right)$ are chosen to be very large. 


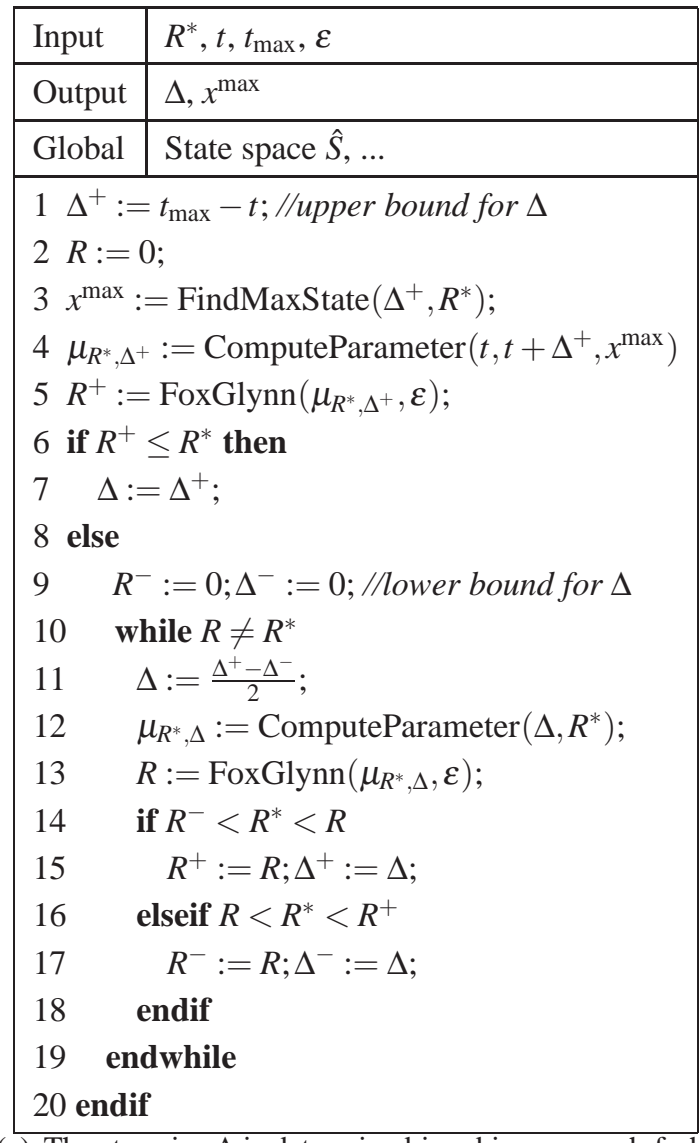

(a) The step size $\Delta$ is determined in a binary-search fashion.

\begin{tabular}{|c|c|}
\hline Input & $t_{0}, t_{\max }, p^{t_{0}}, \varepsilon, R^{*}$ \\
\hline Output & $p^{t_{1}}, p^{t_{2}}, \ldots, p^{t_{\max }}$ \\
\hline Global & State space $\hat{S}, \ldots$ \\
\hline $\begin{array}{ll}1 & t_{\text {cur }}:= \\
2 & \left(\Delta, x^{\mathrm{n}}\right. \\
3 & t_{\text {next }}: \\
4 & \mu:= \\
5 & \text { while } \\
6 \quad i:= \\
7 \quad \text { wh } \\
8 \\
9 \\
9 \\
10 \\
11 \\
12 \quad \text { en } \\
13 \quad t_{c u} \\
14 \quad(\Delta \\
15 \quad t_{n e} \\
16 \quad \mu \\
17 \text { end }\end{array}$ & $\begin{array}{l}t_{0} ; \\
\text { ax }):=\operatorname{Algorithm~} 1\left(R^{*}, t_{\text {cur }}, t_{\max }, \varepsilon\right) ; \\
=t_{\text {cur }}+\Delta ; \\
\text { ComputeParameter }\left(t_{\text {cur }}, t_{\text {next }}, x^{\max }\right) ; \\
t_{\text {cur }} \leq t_{\max } \\
1 \\
\text { le } i \leq R^{*} \\
\text { ompute } v^{(i)}(x) ; / / D T M C \text { probabilities } \\
\text { ompute IPP N probabilities; } \\
\text { Accumulate } \hat{p}^{t_{\text {cur }}}(x) ; / / C T M C \text { probabilities } \\
:=i+1 ; \\
\text { dwhile } \\
:=t_{\text {next }} ; \\
\left.x^{\text {max }}\right):=\text { Algorithm } 1\left(R^{*}, t_{\text {cur }}, t_{\max }, \varepsilon\right) ; \\
:=t_{\text {cur }}+\Delta ; \\
=\text { ComputeParameter }\left(t_{\text {cur }}, t_{\text {next }}, x^{\max }\right) ; \\
\text { hile }\end{array}$ \\
\hline
\end{tabular}

(b) The complete algorithm.

Fig. 1: Algorithms

The function ComputeParameter $\left(t, t+\Delta, x^{\max }\right)$ now computes the integral $\mu_{R^{*}, \Delta}$ using $x^{\max }$. If possible we compute the integral analytically, otherwise we use a numerical integration technique. The function FoxGlynn $(\mu, \varepsilon)$ computes the right truncation point of a homogeneous Poisson process with rate $\mu$ for a given error bound $\varepsilon$, i.e. the value $\hat{R}$ that is the smallest positive integer such that

$$
\sum_{i=0}^{\hat{R}} \frac{\mu^{i}}{i !} e^{-\mu} \geq 1-\varepsilon
$$

For the refinement of the bounds $\Delta^{-}$and $\Delta^{+}$in lines $13-17$ we exploit that $R$ is monotone in $\Delta$.

\subsection{Determining the maximal rates}

The function FindMaxState $\left(\Delta, R^{*}\right)$ in Algorithm 1(a) finds a state $x^{\max }$ such that its exit-rate is greater or equal than the maximal exit-rate $\alpha_{0}\left(x, t^{\prime}\right)=\sum_{j=1}^{m} \alpha_{j}\left(x, t^{\prime}\right)$ over all states $x$ in $S_{t, R^{*}}$. In principal it is enough to find a function $\Lambda\left(t^{\prime}\right)$ with this property, for instance the function $\max _{x \in S_{t, R^{*}}} \sum_{j=1}^{m} \alpha_{j}\left(x, t^{\prime}\right)$, but this function may be hard to determine analytically and it is also not clear how to represent such a function practically in an implementation. Selecting a state $x^{\max }$ and defining $\Lambda\left(t^{\prime}\right)$ to be the exit-rate of this state solves these problems. 
We now present two ways of implementing the function FindMaxState.

a) For this approach we assume that all rate functions increase monotonically in the state variables. This is, for instance, always the case for models from chemical kinetics. We exploit that the change vectors are constant and define for each dimension $k \in\{1, \ldots, n\}$

$$
w_{k}^{\max }:=\max _{j \in\{1, \ldots, m\}} w_{j k}
$$

where $w_{j k}$ is the $k$-th entry of the change vector $w_{j}$. For the set $S_{t, 0}$ we compute, the maximum value for each dimension $k \in\{1, \ldots, n\}$

$$
y_{k}^{\max }:=\max _{y \in S_{t, 0}} y_{k}
$$

We now find the state $x^{\max }$ which is guaranteed to have a higher exit-rate than any state in $S_{t, R^{*}}$ for all time-points in the interval $[t, t+\Delta)$ as follows,

$$
x_{k}^{\max }:=y_{k}^{\max }+R^{*} \cdot w_{k}^{\max } .
$$

It is obvious that the state variables $x_{k}^{\max }$ are upper bounds for the state variables appearing in $S_{t, R^{*}}$. Then, since all rates increase monotonically in the state variables, we have that the exitrate of $x^{\max }=\left(x_{1}^{\max }, \ldots, x_{n}^{\max }\right)$ must be an upper-bound for the exit-rates appearing in $S_{t, R^{*}}$ for all time-points.

b) The first two moments of a Markov population model can be accurately approximated using the method of moments proposed by Engblom [6]. This approximation assumes that the expectations and the (co-)variances change continuously and deterministically in time and it is accurate for most models with rate functions that are at most quadratic in the state variables. We approximate the means $E_{k}\left(t^{\prime}\right):=E\left[X_{k}\left(t^{\prime}\right)\right]$ and the variances $\sigma_{k}^{2}\left(t^{\prime}\right):=\operatorname{VAR}\left[X_{k}\left(t^{\prime}\right)\right]$ for all $k \in\{1, \ldots, n\}$. For each $k$, we determine the time instant $\hat{t} \in[t, t+\Delta)$ at which $E_{k}(\hat{t})+\ell \cdot \sigma_{k}(\hat{t})$ is maximal for some fixed $\ell$. We use this maximum to determine the spread of the distribution, i.e. we assume that the values of $X\left(t^{\prime}\right)$ will stay below $x_{k}^{\max }:=E_{k}(\hat{t})+\ell \cdot \sigma_{k}(\hat{t})$ with high probability. Note that a more detailed approach is to consider the multivariate normal distribution with mean $E\left[X\left(t^{\prime}\right)\right]$ and covariance matrix $\operatorname{COV}\left[X\left(t^{\prime}\right)\right]$. But since the spread of a multivariate normal distribution is difficult to derive in higher dimensions, we simply consider each dimension independently. We now have $x^{\max }=\left(x_{1}^{\max }, \ldots, x_{n}^{\max }\right)$. If during the analysis a state is found which exceeds $x^{\max }$ in one dimension then we repeat our computation with a higher value for $\ell$. To make this approach efficient, $\ell$ has to be chosen in an appropriate way. Our experimental results indicate that for two-dimensional systems the choice $\ell=4$ yields the best results.

\subsection{Complete algorithm}

Our complete algorithm now proceeds as follows (see Algorithm 1(b) . Given an initial distribution $p^{(0)}$ with finite support $S_{0,0}$, a time-bound $t^{\max }$, thresholds $\delta$ and $\varepsilon$, and a desired right truncation point $R^{*}$, we first set $t:=0$. Now we compute a time-step $\Delta$ and the state $x^{\max }$ using Algorithm 1(a) with inputs $R^{*}, t, t^{\max }$, and $\varepsilon$. We then approximate the transient distribution $\hat{p}^{t+\Delta}$ using an on-the-fly version of the bounding approach [2], where the state space is dynamically maintained and states with probability less than $\delta$ are discarded as described above. For the rate function $\Lambda(t)$ we use the exit-rate of state $x^{\max }$. 
When computing DTMC probabilities, we use exact formulas for the first two terms [2] of the sum in Eq. (9) and lower bounds, given by Eq. (10), for the rest. This gives us the approximation $\hat{p}^{t+\Delta}$ with finite support $S_{t+\Delta, 0}$. We now set $t:=t+\Delta$ and repeat the above step with initial distribution $\hat{p}^{t}$ until we have $t=t^{\max }$.

\section{Case Studies}

We implemented the approach outlined in Section 4 in C++ and ran experiments on a $2.4 \mathrm{GHz}$ Linux machine with 4 GB of RAM. We consider a Markov population model that describes a network of chemical reactions. According to the theory of stochastic chemical kinetics [8], the form of the rate function of a reaction depends on how many molecules of each chemical species are needed for one instance of the reaction to occur. The relationship to the volume has been discussed in detail by Wolkenhauer et al. [17]. If no reactants are needed 2 , that is, the reaction is of the form $\emptyset \rightarrow \ldots$ then $\alpha_{j}(x, t)=k_{j} \cdot V(t)$ where $k_{j}$ is a positive constant and $V(t)$ is the volume of the compartment in which the reactions take place. If one molecule is needed (case $S_{i} \rightarrow \ldots$ ) then $\alpha_{j}(x, t)=k_{j} \cdot x_{i}$ where $x_{i}$ is the number of molecules of type $S_{i}$. Thus, in this case, $\alpha_{j}(x, t)$ is independent of time. If two distinct molecules are needed (case $\left.S_{i}+S_{\ell} \rightarrow \ldots\right)$ then $\alpha_{j}(x, t)=\frac{k_{j}}{V(t)} \cdot x_{i} \cdot x_{\ell}$.

All these theoretical considerations are based on the assumption that the chemical reactions are elementary, that is, they are not a combination of several reactions. Our example may contain nonelementary reactions and thus a realistic biological model may contain different volume dependencies. But since the focus of the paper is on the numerical algorithm, we do not aim for an accurate biological description here.

We conduct experiments with two reaction networks. The first one is a simple gene expression (described in Ex. 11). The second one is a gene regulatory network, called the exclusive switch [12]. It consists of two genes with a common promotor region. Each of the two gene products $P_{1}$ and $P_{2}$ inhibits the expression of the other product if a molecule is bound to the promotor region. More precisely, if the promotor region is free, molecules of both types $P_{1}$ and $P_{2}$ are produced. If a molecule of type $P_{1}$ is bound to the promotor region, only molecules of type $P_{1}$ are produced. If a molecule of type $P_{2}$ is bound to the promotor region, only molecules of type $P_{2}$ are produced. No other configuration of the promotor region exists. The probability distribution of the exclusive switch is bistable which means that after a certain amount of time, the probability mass concentrates on two distinct regions in the state space. The system has five chemical species of which two have an infinite range, namely $P_{1}$ and $P_{2}$. We define the transition classes $\tau_{j}=\left(G_{j}, w_{j}, \alpha_{j}\right), j \in\{1, \ldots, 10\}$ as follows.

- For $j \in\{1,2\}$ we describe production of $P_{j}$ by $G_{j}=\left\{x \in \mathbb{N}^{5} \mid x_{3}>0\right\}, w_{j}=e_{j}$, and $\alpha_{j}(x, t)=$ $0.5 \cdot x_{3}$. Here, $x_{3}$ denotes the number of unbound DNA molecules which is either zero or one and the vector $e_{j}$ is such that all its entries are zero except the $j$-th entry which is one.

- We describe degradation of $P_{j}$ by $G_{j+2}=\left\{x \in \mathbb{N}^{5} \mid x_{j}>0\right\}, w_{j+2}=-e_{j}$, and $\alpha_{j+2}(x, t)=0.005 \cdot x_{j}$. Here, $x_{j}$ denotes the number of $P_{j}$ molecules.

- We model the binding of $P_{j}$ to the promotor as $G_{j+4}=\left\{x \in \mathbb{N}^{5} \mid x_{3}>0, x_{j}>0\right\}, w_{j+4}=-e_{j}-$ $e_{3}+e_{j+3}$, and $\alpha_{j+4}(x, t)=\left(0.1-\frac{0.05}{3600} \cdot t\right) \cdot x_{j} \cdot x_{3}$ for $t \leq 3600$. Here, $x_{j+3}$ is one if a molecule of type $P_{j}$ if bound to the promotor region and zero otherwise.

\footnotetext{
${ }^{2}$ Typically, reactions requiring no reactants are used in the case of open systems where it is assumed that the reaction is always possible at a constant rate and the reactant population is not explicitly modelled.
} 


\begin{tabular}{|c|c|c|c|c|c|c|c|}
\hline Case study & $\begin{array}{l}\text { FindMaxState } \\
\text { implementation }\end{array}$ & $R^{*}$ & $\begin{array}{l}\text { Total } \\
\text { error }\end{array}$ & Ex. time & $|S|$ & $\min _{\%}$ & Poisson $\%$ \\
\hline \multirow{8}{*}{$\begin{array}{c}\text { Gene } \\
\text { expression }\end{array}$} & \multirow{4}{*}{ method a) } & 5 & $4.69 \cdot 10^{-4}$ & $14 \mathrm{~min}$ & \multirow{4}{*}{33962} & 95 & 5 \\
\hline & & 10 & $1.33 \cdot 10^{-2}$ & $10 \mathrm{~min}$ & & 78 & 22 \\
\hline & & 15 & $2.24 \cdot 10^{-2}$ & $5 \mathrm{~min}$ & & 64 & 36 \\
\hline & & 20 & $9.92 \cdot 10^{-2}$ & $3 \mathrm{~min}$ & & 41 & 59 \\
\hline & \multirow{4}{*}{ method b) } & 5 & $4.78 \cdot 10^{-4}$ & $27 \mathrm{~min}$ & \multirow{4}{*}{33130} & 95 & 5 \\
\hline & & 10 & $9.63 \cdot 10^{-3}$ & $14 \min$ & & 77 & 23 \\
\hline & & 15 & $4.08 \cdot 10^{-2}$ & $10 \mathrm{~min}$ & & 58 & 42 \\
\hline & & 20 & $7.73 \cdot 10^{-2}$ & $7 \mathrm{~min}$ & & 41 & 59 \\
\hline \multirow{8}{*}{$\begin{array}{l}\text { Exclusive } \\
\text { switch }\end{array}$} & \multirow{4}{*}{ method a) } & 5 & $2.38 \cdot 10^{-6}$ & $21 \mathrm{~min}$ & \multirow{4}{*}{1740} & 80 & 20 \\
\hline & & 10 & $1.63 \cdot 10^{-5}$ & $29 \mathrm{~min}$ & & 75 & 25 \\
\hline & & 15 & $2.51 \cdot 10^{-5}$ & $68 \mathrm{~min}$ & & 47 & 53 \\
\hline & & 20 & $3.32 \cdot 10^{-5}$ & $2 \mathrm{~h}$ & & 38 & 62 \\
\hline & \multirow{4}{*}{ method b) } & 5 & $3.56 \cdot 10^{-6}$ & $17 \mathrm{~h}$ & \multirow{4}{*}{1752} & 89 & 11 \\
\hline & & 10 & $1.55 \cdot 10^{-4}$ & $3 \mathrm{~h}$ & & 78 & 22 \\
\hline & & 15 & $6.51 \cdot 10^{-4}$ & $1.5 \mathrm{~h}$ & & 59 & 41 \\
\hline & & 20 & $1.71 \cdot 10^{-3}$ & $1 \mathrm{~h}$ & & 42 & 58 \\
\hline
\end{tabular}

Table 1: Results of the analysis of case studies.

- For unbinding of $P_{j}$ we define $G_{j+6}=\left\{x \in \mathbb{N}^{5} \mid x_{j+3}>0\right\}, w_{j+6}=e_{j}+e_{3}-e_{j+3}$, and $\alpha_{j+6}(x, t)=$ $0.005 \cdot x_{j+3}$.

- Finally, we have production of $P_{j}$ if a molecule of type $P_{j}$ is bound to the promotor, i.e., $G_{j+8}=$ $\left\{x \in \mathbb{N}^{5} \mid x_{j+3}>0\right\}, w_{j+8}=e_{j}$, and $\alpha_{j+8}(x, t)=0.5 \cdot x_{j+3}$.

Note that only the rate functions $\alpha_{5}$ and $\alpha_{6}$, which denote the binding of a protein to the promotor region, are time-dependent. This is intuitively clear since if the cell volume grows it becomes less likely that a protein molecule is located close to the promotor region. We started the system at time $t=0$ in state $(0,0,1,0,0)$ with probability one and considered a time horizon of $t=3600$. For the simple gene expression system (Example 10 we started at time $t=0$ in state $(0,0)$ and considered the same time horizon. Table 1 contains the results of our experiments. The first column refers to the system under study and the second one shows the variation used to implement the method FindMaxState which we suggest in Section 4.2. The third column lists the different values for right truncation point $R^{*}$. We list the total error at time $t_{\max }$ in the fourth column (see Eq. (11)). Program execution time is given in the fifth column and the sixth column with heading $|S|$ contains the maximal size of the set $S_{t, R^{*}}$ that we considered during the analysis. The next two columns describe the percentage of the total probability loss due to the bounding approach $\left(\min _{\%}\right)$ and due to the truncation of the infinite sum in Eq. (5) (Poisson $\%$ ). The two percentages in one row do not sum up to one since we store only states that have significant probability (w.r.t threshold $\delta$ ), which is the third error source. However, this lost portion is negligible for the two systems that we consider. For our implementation we kept the input $\varepsilon=10^{-10}$ of Algorithm 1(a) fixed. 


\subsection{Discussion}

We now discuss the effect of the different input parameters on the performance of our algorithm and start with the implementation of the method to approximate $x^{\max }$. For both systems the method " $\mathrm{b}$ " is less effective than method "a" (see Section 4.2). Method "b" gives larger uniformization rates than method "a", which leads to slower execution times. Notice that the execution time grows when we use method "a" for the exclusive switch system when we choose the larger values for $R^{*}$. This is due to the fact that it always finds a state $x^{\max }$ without taking expectations and covariances into consideration. This results in large over-approximations for such a bi-stable system. The effect of the choice between methods "a" and " $b$ " on the accuracy is not completely clear, both methods provide the same order of the probability loss for the simple gene expression system. For the second case study method "a" provides tighter error bounds for larger values of $R^{*}$.

In the Table 1 we show results obtained with $\delta=10^{-15}$. Naturally, choosing a lower threshold results in larger execution times but one can gain a deeper exploration of the state space. This fact can also be used to obtain a coarse solution for certain system by setting $\delta=10^{-5}$, for instance.

The effect of the choice of $R^{*}$ is most interesting. Choosing a larger value for $R^{*}$ means that more summands on the right-hand side of Eq. (10) have to be approximated using the bounding approach. This decreases the accuracy of the algorithm since the larger time steps $\Delta$ are conducted and one obtain coarse approximation. However it reduces the running time since $t_{\max }$ can be covered using fewer iterations. Notice that the percentage of the probability loss due to truncation of the infinite sum in Eq. (5) grows when $R^{*}$ is chosen to be large. The reason is that we compute only first 3 exact terms in the sum and remaining terms are approximations. Thus the choice of $R^{*}$ determines the compromise between running time and accuracy.

\section{Conclusion}

We have presented an algorithm for the numerical approximation of transient distributions for infinite time-inhomogeneous Markov population models with unbounded rates. Our algorithm provides a strict lower bound for this transient distribution. There is a trade-off between the tightness of the bound and the performance of the algorithm, both in terms of computation time and required memory.

As future work, we will investigate the relationship between the parameters of our approach (truncation point, the significance threshold $\delta$, the method by which we determine the rate of the Poisson process), the accuracy and the running time of the algorithm more closely. For this we will consider Markov population models with different structures and dynamics.

\section{References}

[1] A. Arkin, J. Ross \& H. H. McAdams (1998): Stochastic Kinetic Analysis of Developmental Pathway Bifurcation in Phage $\lambda$-Infected Escherichia coli Cells. Genetics 149, pp. 1633-1648.

[2] M. Arns, P. Buchholz \& A. Panchenko (2010): On the numerical Analysis of Inhomogeneous Continuous Time Markov Chains. INFORMS Journal on Computing 22, pp. 416-432, doi:10.1287/i joc.1090 . 0357.

[3] G. Ciardo (1995): Discrete-time Markovian stochastic Petri nets. Kluwer.

[4] F. Didier, T. A. Henzinger, M. Mateescu \& V. Wolf (2009): Fast Adaptive Uniformization of the Chemical Master Equation. In: Proc. of HIBI. pp. 118-127, doi:10.1109/HiBi.2009.23. 
[5] N.M. van Dijk (1992): Uniformization for nonhomogeneous Markov chains. Operations research letters 12(5), pp. 283-291, doi:10.1016/0167-6377(92) 90086-I.

[6] S. Engblom (2006): Computing the moments of high dimensional solutions of the master equation. Appl. Math. Comput. 180, pp. 498-515, doi:10.1016/j.amc.2005.12.032.

[7] D. T. Gillespie (1976): A General Method for Numerically Simulating the Time Evolution of Coupled Chemical Reactions. J. Comput. Phys. 22, pp. 403-434, doi:10.1016/0021-9991 (76) 90041-3.

[8] D. T. Gillespie (1977): Exact Stochastic Simulation of Coupled Chemical Reactions. J. Phys. Chem. 81(25), pp. 2340-2361, doi:10.1021/j100540a008.

[9] W. K. Grassmann (1990): Computational methods in probability theory. In D. P. Heyman \& M. J. Sobel, editors: Stochastic Models, chapter 5. Handbooks in Operations Research and Management Science 2, Elsevier, pp. 199-254, doi:10.1016/S0927-0507 (05) 80169-0.

[10] A. Jensen (1953): Markoff chains as an aid in the study of Markoff processes. Skandinavisk Aktuarietidskrift 36, pp. 87-91.

[11] J. F. C. Kingman (1969): Markov Population Processes. Journal of Applied Probability 6(1), pp. 1-16.

[12] A. Loinger, A. Lipshtat, N. Q. Balaban \& O. Biham (2007): Stochastic simulations of genetic switch systems. Phys. Rev. E 75(2), p. 021904, doi:10.1103/PhysRevE.75.021904.

[13] A. P. A. van Moorsel \& K. Wolter (1998): Numerical Solution of Non-Homogeneous Markov Processes through Uniformization. In: Proc. of the European Simulation Multiconference - Simulation. SCS Europe, pp. 710-717.

[14] E. de Souza e Silva \& P. M. Ochoa (1992): State Space Exploration in Markov Models. In: SIGMETRICS. pp. 152-166, doi:10.1145/133057.133100.

[15] W. J. Stewart (1995): Introduction to the Numerical Solution of Markov Chains. Princeton University Press.

[16] M. Thattai \& A. van Oudenaarden (2001): Intrinsic noise in gene regulatory networks. PNAS, USA 98(15), pp. 8614-8619, doi:10.1073/pnas.151588598.

[17] O. Wolkenhauer, M. Ullah, W. Kolch \& K. Cho (2004): Modeling and Simulation of Intracellular Dynamics: Choosing an Appropriate Framework. IEEE Transactions on NanoBioscience 3(3), pp. 200-207, doi:10 . $1109 /$ TNB.2004.833694. 